\title{
The Capacity of Vehicular Ad Hoc Networks with Infrastructure
}

\author{
Mohammad Nekoui, Ali Eslami, Hossein Pishro-Nik \\ Electrical and Computer Engineering Department \\ University of Massachusetts, Amherst
}

\begin{abstract}
In this paper, we initiate a framework to address the capacity scaling trends in Vehicular Ad Hoc Networks (VANET)s with arbitrary topologies. Towards this end we utilize the conventional definition of transport capacity in which destination nodes are chosen at random by the source nodes. Also, to get more VANET-specific, we set up a new variation of transport capacity in which the destination nodes are again chosen at random, but this time are within a distance $d$ of the source node which is called the distance-limited capacity. Emergency and accident avoidance scenarios are just, some of the direct applications of distance-limited capacity. Moving on further, we study the effect of infrastructure node deployment in the capacity analysis of VANETs. We've initiated this trend with our focus on the distance-limited capacity of a single road VANET. Using analytical expressions we show that exploiting any number of infrastructure nodes beyond a certain amount, enhances the achievable capacity.
\end{abstract}

\section{INTRODUCTION}

The emerging field of Vehicular Ad hoc Networks (VANET)s has attracted the attention of many researchers in recent years. Due to the high priority ascribed to safety and emergency applications in transportation systems, the VANET frontier has evolved into a dynamic research field. VANETs use Inter-Vehicle Communication (IVC) that is an important component of the Intelligent Transportation System (ITS) architecture. VANET will enable a wide range of novel applications such as accident avoidance messaging, congestion sensing, traffic metering, and general information services (e.g., Internet access). The allocation of $75 \mathrm{MHz}$ in the $5.9 \mathrm{GHz}$ band for Dedicated Short Range Communications (DSRC) may also enable future delivery of rich media content to vehicles at short to medium ranges via both inter-vehicle and road-vehicle communications. The soon-to-be-launched Vehicle Infrastructure Integration (VII) in the U.S. envisions that a future vehicle will be equipped with On-Board Equipment (OBE), and the communication infrastructure will include Roadside Equipment (RSE)s that is deployed at strategic locations along the roadside.

Despite the increasing amount of research on VANETs, the capacity scaling analysis of such networks is still in its infancy. In [1], some expressions were derived to assess the asymptotic behavior of VANETs in single road and grid topology cases. As it was seen there, even a single isolated rural area road can potentially have every possible capacity scaling just based on its path geometry. Such a phenomenon was not observed in ordinary analysis of wireless networks. Thus, there is a need to categorize roads based on their geometric properties.

Another issue is the effect of vehicle mobility in VANET's capacity scaling. There is some interesting literature on the effects of mobility on the capacity of wireless networks [4-8]. In these analyses it is usually assumed that there is high delay tolerance, nodes have huge buffer sizes, and the network topology changes over time-scale of packet delivery. Indeed none of the assumptions hold in VANETs. For example, emergency and safety-related messages are extremely delay sensitive in VANETs. More importantly, a very common assumption in the literature is that the nodes move independently of each other. This assumption by no means holds in VANETs. In fact our results suggests unlike the existing literature, mobility does not improve capacity scaling in VANETs. This is in contrast with the previous conception on mobility and capacity.

Finally note that, in the study of transport capacity it is usually assumed that each node has a random destination chosen uniformly from the available nodes in the network. In [2] we focused on how to determine the capacity bounds and its achievability for VANETs' distance-limited capacity. In distance-limited communications each vehicle is only interested to communicate with other vehicles which reside in a predetermined area of its vicinity. This definition, is of course an outcome of the specific application of VANETs in emergency situations and significantly affects the scaling laws for throughput.

In this paper we extend the study of VANETs' scaling laws towards more general transportation systems topologies. Also we provide elementary results regarding the deployment of infrastructure nodes, termed as Road Side Units (RSU)s, in single road VANET deployment. For simplicity, we adopt the protocol model introduced in [13]. The methodology can be extended to other models as well. The conventional transport capacity of a VANET with $n$ nodes is shown by $\Lambda(n)$. It is assumed that each node has a communication radius $r_{t}$. 


\section{Formulation AND PRELIMINARIES}

We show a lane on a road by a parameterized smooth continuous curve $X_{n}(s)=\left(x_{n}(s), y_{n}(s)\right), s \in[0,1]$ on the plane. The length of each section of the curve is obtained using the Hausdorff one-dimensional measure [9]. The subscript $n$ shows the number of vehicles on the road. The curve shows the trajectory of the road. $X_{n}(0)$ is the beginning of the lane and $X_{n}(1)$ shows the end. It is assumed a road can intersect itself only a finite number of times. Multi-lane roads are indicated by several parallel curves. A transportation network usually consists of several roads. The density, $k_{n}(s)$, of a road is defined by the average number of vehicles per unit length at point $X_{n}(s)$. At any part of the road, the density of cars is assumed to be a bounded positive number as in reality the density is limited by the physical size of cars. In this paper, for simplicity we assume $k_{n}(s)=k$, for all $s \in[0,1]$ in all proofs. However, the results are easily extendable to the general case. For transportation networks consisting of several roads, the values of densities are chosen in a way that the flow conservation principle is satisfied at the intersections.

The mobility model for vehicles is an important factor in vehicular ad hoc networks. It is assumed that the movements of vehicles on the roads follow a stationary stochastic process. Note that in VANETs, the vehicles do not move independently from each other. However, it has been observed that at any time $t$, the positions of vehicles can be modeled based on a Poisson process on the road, thus the spacing between them has exponential distribution [10-12]. In this paper we follow this assumption, however, it can be shown that the results hold for more general mobility models that satisfy some specific conditions. To define our model rigorously, we extend the lane $X_{n}(s)$ from both ends to infinity. Then, we place a Poisson point process with density $k$ on the extended curve. Any point of the process will correspond to a vehicle. At time $t=0$, all vehicles on the same lane will choose a common speed $v \in\left[0, v_{\max }\right]$ uniformly at random. It is assumed that $v_{\max }$ is a fixed and bounded real number. It is assumed that the vehicles do not change their speed. Thus, at any time $t$, the positions of vehicles is still a Poisson process. Since we assume Poisson distribution and are interested in scaling laws, we can often combine parallel lanes to obtain one curve whose density is given by the summation of densities, i.e, $k=k_{1}+k_{2}+\ldots+k_{l}$ to simplify the analysis. However, it is important to note that this is possible only when we are providing macroscopic analysis, otherwise we need to consider each lane separately and account for the interactions between lanes. We assume $B(X, r)$ is the closed ball with radius $r$ centered at $X$ in $\mathbb{R}^{2}$. Also, $C(X, r)$ is the circle with radius $r$ centered at $X$.

We consider transportation networks that consist of $n$ cars equipped with OBEs. We are interested in the fundamental limits of these networks as $n$ grows large for general road topologies. Since the density of cars is a bounded positive number, to have a large number of nodes, the total lengths of the roads are assumed to be large, $L=\frac{n}{k}=\Theta(n)$.

We also make the assumption that the roads are not highly dense on the plane. We call this the sparseness condition. To make this rigorous, for any point $Y \in \mathbb{R}^{2}$, let $l(Y, r)$ be the Hausdorff one-dimensional measure (combined length) of the sections of the roads inside $B(Y, r)$. It is assumed that $l\left(Y, \alpha r_{t}\right)=O\left(r_{t}\right)$ for all $Y \in \mathbb{R}^{2}$ and any constant $\alpha>0$. For any point $X$ on a road let $n(X)$ be the number of times that $C\left(X, r_{t}\right)$ intersects with the road curves. It is assumed that $n(X)$ is bounded. Let $A_{r_{t}}$ be the sections of the roads consisting of points with $n(X)>2$. We say that the road system is sparse if the combined length of $A_{r_{t}}$ is $o(L)$. If $n(X) \leq 2$ for all points on the roads, then the system is said to be highly sparse. At any intersection, it is assumed that only a bounded number of roads can intersect with each other.

\section{RESUlts AND Discussions}

Before stating the results we need a simple lemma. This lemma is used in the proofs of the capacity results.

Lemma 1. Consider a transportation network that consists of $u$ single roads with lengths $l_{1}, l_{2}, \ldots, l_{u}$. Suppose that we divide the roads to sections of lengths $\beta r_{t}$, where $\beta$ is a constant. We can place these sections into a bounded finite number of non-interfering groups.

This result states that we can schedule parallel transmissions in the network as long as the transmissions belong to different groups. This is a standard method used to obtain lower bound on the capacity of wireless networks. The lemma can be proved using graph coloring.

\section{A. Transport Capacity of General Transportation Networks}

Here we discuss a general scenario that includes downtown grids as a special case. Figure 1 shows a typical downtown area. We provide a general result on the capacity of vehicular ad hac networks. In particular, we define the concept of street connectivity. This is somewhat similar to edge-connectivity in Graph Theory. Remember, the edge connectivity shows the size of the minimum cut in a graph. It also relates to the number of disjoint path that exists between two nodes.

Consider two vehicles $C_{1}$ and $C_{2}$ located on the roads $R_{1}$ and $R_{2}$ respectively. Two different paths from $C_{1}$ to $C_{2}$ are called street-disjoint if they do not intersect with each other except on $R_{1}$ and $R_{2}$. We say that a transportation network has street connectivity $m$ if there are at least $m$ street disjoint paths between any two points in the network. Although it is very similar to edge-connectivity in Graph Theory, the street connectivity is affected by both geometrical and the graph 
theoretical properties of the system. In particular, using the assumptions such as sparseness and network connectivity, we show that the street connectivity cannot grow faster than $\Theta\left(\sqrt{\frac{n}{\ln n}}\right)$. Note that in Graph Theory, the edge-connectivity can grow as fast as $\Theta(n)$. We point out the following two lemmas before stating the main result.

Lemma 2. Consider a transportation network that consists of $u$ single roads. Let $\mathcal{L}$ be the subset of $\mathbb{R}^{2}$ containing the road curves and $H($.$) be the Hausdorff one-dimensional$ measure. Let $A_{1}, A_{2}, \ldots, A_{i} \subseteq \mathcal{L}$ be sections of the roads satisfying the following property: For all $I \in\{1,2, \ldots, i\}$ with $|I|>c$, we have $\bigcap_{j \in I} A_{j}=\emptyset$. Then we have

$$
\sum_{j=1}^{i} H\left(A_{j}\right) \leq c H\left(\bigcup_{j=1}^{i} A_{j}\right) .
$$

Lemma 3. Consider a transportation network with street connectivity $m$ that consists of $u$ single roads with lengths $l_{1}, l_{2}, \ldots, l_{u}$. Assume that $\epsilon<\frac{1_{i}}{l_{j}}<\gamma$ for some fixed constant numbers $\epsilon$ and $\gamma$ and for any $i, j \in\{1,2, \ldots, u\}$. Then we have $m=o\left(\sqrt{\frac{n}{\ln n}}\right)$.

We now can state the following theorem on transport capacity of general transportation networks.

Theorem 1. Consider a transportation network with street connectivity $m$ that consists of $u$ single roads with lengths $l_{1}, l_{2}, \ldots, l_{u}$. Assume that $\epsilon<\frac{l_{i}}{l_{j}}<\gamma$ for some fixed constant numbers $\epsilon$ and $\gamma$ and for any $i, j \in\{1,2, \ldots, u\}$. The transport capacity of the corresponding VANET is $\Omega\left(\frac{m}{n}\right)$.

The above theorem simply states that the capacity is determined by the street connectivity of the transportation network. Note that when the stated assumptions such as sparseness and network connectivity hold, it can be shown that the street connectivity cannot grow faster than $\Theta\left(\sqrt{\frac{n}{\ln n}}\right)$. Thus, the capacity does not have to grow faster than $\Theta\left(\sqrt{\frac{1}{n \ln n}}\right)$.

\section{B. Effects of RSUs}

So far we have assumed there is no infrastructure in the network. A natural question is how the infrastructure affects the network capacity. There are some work in the literature discussing the capacity of wireless networks with infrastructure, see for example [3]. Here we consider a similar scenario in which the RSUs do not generate new information and only serve to help the communication between vehicles. Suppose that $R(n)$ is the number of RSUs in the system. It is assumed that the nodes can communicate with each other using a channel with a bounded bandwidth $W_{1}<\infty$, and they can communicate with the RSUs using a channel with a bounded bandwidth $W_{2}<\infty$ that does not interfere with $W_{1}$. Note that, for this case, we consider distance-limited

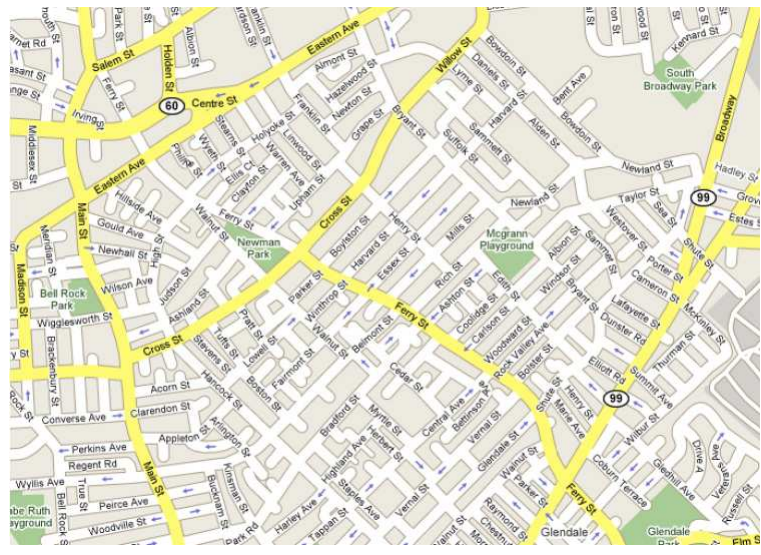

Fig. 1. A typical urban area. This is the map of some part of Boston taken from Google map.

capacity, where nodes can communicate with other nodes which are within a certain range, $d(n)$, of their vicinity.

Theorem 2. Assume the sparseness condition is satisfied. Assume that $r_{t}(n)=\Theta(\ln n)$. Let $\Lambda_{d}^{R}(n)$ be the distancelimited capacity of a single road $X(s), s \in[0,1]$ with $R(n)$ RSUs.

- If $d(n)=\Omega\left(r_{t}(n)\right)$ and $R(n) d(n)=O(n)$ and $R(n)=O\left(\frac{n}{\ln n}\right)$, then $\Lambda_{d}^{R}(n)=\Theta\left(\frac{1}{d}\right)$.

- If $d(n)=O\left(r_{t}(n)\right)$ or $R(n)=\Omega\left(\frac{n}{\ln n}\right)$, then $\Lambda_{d}^{R}(n)=$ $\Theta\left(\frac{1}{\ln n}\right)$.

- If $R(n) d(n)=\Omega(n)$, and $d(n)=\Omega\left(r_{t}(n)\right)$, and $R(n)=O\left(\frac{n}{\ln n}\right)$, then $\Lambda_{d}^{R}(n)=\Theta\left(\frac{R(n)}{n}\right)$.

The above formula simply states that, when the number of RSUs is so small that it's unlikely that there exists at least one RSU in any section of length $d(n)$ of the road, the capacity is virtually the same as the pure ad hoc case. Moreover by increasing the number of RSUs, so that we have at least one RSU in each section, the capacity increases almost linearly with the number of RSUs. Increasing the number of RSUs beyond a certain amount is of no use as capacity is limited by the number of simultaneous transmissions each of them can handle. In figure 2, we have illustrated the capacity regions for a single road VANET with $R(n)$ RSUs.

\section{PROOFS AND ANALYSIS}

Here we outline the basis of the proofs for lemma 1 and the aforementioned theorems.

\section{Proof of Lemma 1}

Proof: Construct the interference graph $G$ in the following way. Any section of any road will be a vertex in $G$. Two vertices in $G$ are connected to each other if the distance between the two corresponding sections is less than or equal to $r_{t}$. The requirements of sparseness condition guarantee that the maximum degree of $G$ is a finite bounded number. 


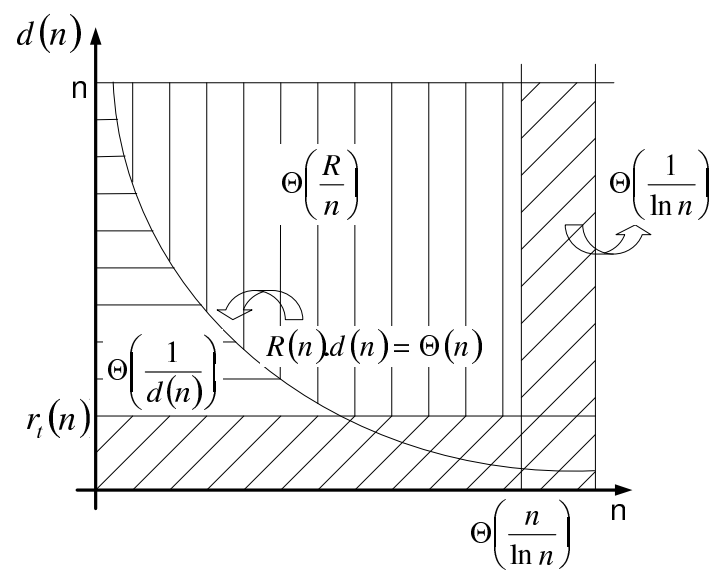

Fig. 2. Capacity regions for VANETs on a single road with $R(n)$ RSUs.

Thus the chromatic index of $G$ is a bounded number, too. We conclude that the vertices of $G$ can be colored using a finite number of colors so that no two vertices with the same color are adjacent. Each color group represents the sections of the road belonging to one of the non-interfering groups. The number of groups is bounded because the chromatic index is bounded.

\section{Proof of Lemma 2}

Proof: Since the set $\mathcal{L}$ is bounded it is completely inside a square $S_{0}$ with side $b$. Define $s h_{b}():. \mathbb{R}^{2} \mapsto \mathbb{R}^{2}$, as $\operatorname{sh}_{b}(x, y)=(x+b, y)$. For any measurable set $E \in \mathbb{R}^{2}$, we have $H\left(\operatorname{sh}_{b}(E)\right)=H(E)$. Define

$$
\begin{aligned}
& \mathcal{L}^{1}=\bigcup_{j=1}^{i} A_{j} \\
& \mathcal{L}^{2}=\operatorname{sh}_{b}\left(\mathcal{L}^{1}\right) \\
& \mathcal{L}^{3}=\operatorname{sh}_{b}\left(\mathcal{L}^{2}\right)=s h_{b}^{2}\left(\mathcal{L}^{1}\right) \\
& \cdot \\
& \cdot \\
& \cdot \\
& \mathcal{L}^{c}=\operatorname{sh}_{b}^{c-1}(\mathcal{L}) .
\end{aligned}
$$

Now define functions $f_{j}: \mathbb{R}^{2} \mapsto \mathbb{R}^{2}, j \in\{1,2, \ldots, i\}$ in the following way. $f_{j}(x, y)=(x+t b, y)$, where $x$ belongs to $t$ of the sets $A_{1}, A_{2}, \ldots, A_{j-1}$. We conclude from the assumptions of the lemma that

$$
\bigcup_{j=1}^{i} f_{j}\left(A_{j}\right) \subseteq \bigcup_{k=1}^{c} \mathcal{L}^{k}
$$

For all $j \neq k$, we have $f_{j}\left(A_{j}\right) \cap f_{k}\left(A_{k}\right)=\emptyset$. Moreover, since $i$ is a finite number, we have $H\left(f_{j}\left(A_{j}\right)\right)=H\left(A_{j}\right)$. We conclude

$$
H\left(\bigcup_{j=1}^{i} f_{j}\left(A_{j}\right)\right)=\sum_{j=1}^{i} H\left(f_{j}\left(A_{j}\right)\right)=\sum_{j=1}^{i} H\left(A_{j}\right) .
$$

Combining Equations (2) and (3), we obtain

$$
\sum_{j=1}^{i} H\left(A_{j}\right) \leq H\left(\bigcup_{k=1}^{c} \mathcal{L}^{k}\right)=c H\left(\mathcal{L}^{1}\right)=c H\left(\bigcup_{j=1}^{i} A_{j}\right)
$$

\section{Proof of Lemma 3}

Proof: Since the system has street connectivity $m$, we conclude that each road intersects with at least $m$ other roads and also $u \geq m$. Thus the number of intersections in the system is $i(n) \geq m^{2}$. Number the intersections in the system from 1 to $i(n)$, Remember that for any point $X$ on a road $n(X)$ is the number of times that $C\left(X, r_{t}\right)$ intersects with the road curves. For any point $X$ on a road, we say that $X$ is contradicting point if $n(X)>2$. Note that any intersection will create a section of length at least $\Theta\left(r_{t}\right)$ consisting of contradicting points. For the $j$ th intersection let $A_{j}$ be the sections of the road consisting of the contradicting points due to the intersection. Then $A_{j}=\Omega\left(r_{t}\right)$. since we assume that $n(X)$ is bounded at any point in the network, there exists a constant $c>0$ such that $n(x)<c$ for all $X$. That means that for all $I \in\{1,2, \ldots, i(n)\}$ with $|I|>c$, we have $\bigcap_{j \in I} A_{j}=\emptyset$. Now using Lemma 2 we conclude

$$
\sum_{j=1}^{i(n)} H\left(A_{j}\right) \leq c H\left(\bigcup_{j=1}^{i(n)} A_{j}\right) .
$$

However, from the sparseness condition we conclude that $H\left(\bigcup_{j=1}^{i(n)} A_{j}\right)=o(n)$. Thus, we have

$$
\sum_{j=1}^{i(n)} H\left(A_{j}\right)=o(n) .
$$

On the other hand, we have $\sum_{j=1}^{i(n)} H\left(A_{j}\right) \geq i(n) \Theta\left(r_{t}\right)$. Connectivity of the network implies that $r_{t}(n)=\Omega(\ln n)$, thus we have

$$
\sum_{j=1}^{i(n)} H\left(A_{j}\right)=\Omega\left(m^{2} \ln n\right) .
$$

combining Equations (6) and (7), we conclude $m=$ $o\left(\sqrt{\frac{n}{\ln n}}\right)$. 


\section{Proof of Theorem 1}

Proof: We provide a routing strategy that achieves $\Theta\left(\frac{m}{n}\right)$ per node throughput. First note that by the definitions of street connectivity we have $u \geq m$. The algorithm works in the following way. First, as usual, divide the road into sections of length $\frac{r_{t}}{2}=\Theta\left(\frac{\ln n}{2 k}\right)$. Using the coloring Lemma 1 , we can divide the sections into a finite number of noninterfering groups. For any source-destination pair consider a set of $m$ disjoint street paths. Choose one of the paths at random and use multi-hop communications between the sections to send the messages from the sender to the receiver node. We prove we can achieve $\Theta\left(\frac{m}{n}\right)$ per node throughput using this algorithm.

At any time, there are $n(1+o(1))$ nodes in the network. Order these nodes randomly and call them node1, node 2, $\ldots$, and node $n$. Define Boolean random variables $X_{i j}$ in the following way. $X_{i j}=1$ if and only if the routing path starting at node $j$ uses at least one section of road $i$ in the network. We claim Prob $\left\{X_{i j}=1\right\}=O\left(\frac{1}{m}\right)$. Indeed, if the node $j$ or its destination are on the $\operatorname{road} i$, then $X_{i j}=1$ with probability one. This event occurs with probability $\Theta\left(\frac{1}{u}\right)$. On the other hand, if node $j$ and its destination are not on the $\operatorname{road} i$, then Prob $\left\{X_{i j}=1\right\} \leq \frac{1}{m}$, because the road $i$ can be in at most one of the $m$ street-disjoint paths from node $j$ to its destination. Thus,

$$
\operatorname{Prob}\left\{X_{i j}=1\right\} \leq \Theta\left(\frac{1}{u}\right)+\frac{1}{m}=O\left(\frac{1}{m}\right),
$$

where we used $u \geq m$. Thus we conclude that $E X_{i j}=$ $\rho(1+o(1)) \frac{1}{m}$, where $\rho$ is a positive constant number. Define the random variables $X_{i}$ as

$$
X_{i}=\sum_{j=1}^{n} X_{i j}
$$

We have $E X_{i}=\rho(1+o(1)) \frac{n}{m}$. Define the event $E_{i}$ as $\left\{X_{i} \leq\left(1+\frac{1}{\rho}\right) E X_{i}\right\}$. We show that the event $\bigcap_{i=1}^{u} E_{i}$ occurs with high probability. Define $F_{i}=E_{i}^{c}$. Note that $X_{i j}$ are i.i.d random variables. Thus we can construct a martingale and apply the Azuma's inequality. We conclude

$$
\begin{aligned}
\operatorname{Prob}\left\{F_{i}\right\} & \leq \operatorname{Prob}\left\{\left|X_{i}-E X_{i}\right|>\frac{1}{\rho} E X_{i}\right\} \\
& <\exp \left(-\frac{(E X)^{2}}{\rho^{2} u}\right) \\
& =\exp \left(-\frac{n^{2}(1+o(1))}{u m^{2}}\right) \\
& \leq \exp \left(-\frac{n^{2}}{2 u m^{2}}\right) .
\end{aligned}
$$

By Lemma 3, we can write $m=\sqrt{\frac{n}{\ln n}} \frac{1}{w(n)}$, where $w(n) \rightarrow \infty$ as $n$ goes to infinity. Thus we conclude

$$
\begin{aligned}
\operatorname{Prob}\left\{\bigcup_{i=1}^{u} F_{i}\right\} & \leq \exp \left(-\frac{n^{2}}{2 u m^{2}}\right) \\
& =\exp \left(-\frac{n \ln n w^{2}(n)}{2 u}\right) \\
& \leq n^{-\frac{w^{2}(n)}{2}}=o(1) .
\end{aligned}
$$

Since we have $\operatorname{Prob}\left\{\bigcap_{i=1}^{u} E_{i}\right\}=1-\operatorname{Prob}\left\{\bigcup_{i=1}^{u} F_{i}\right\}$, we conclude $\bigcap_{i=1}^{u} E_{i}$ occurs with high probability. This shows that each section of the roads has to support at most $O\left(\frac{n}{m}\right)$ path, and thus the throughput $\Theta\left(\frac{m}{n}\right)$ is achievable.

\section{Proof of Theorem 2}

Proof:

The first of the three listed items, represents the case where we are concerned with multi-hop communications and the fact that we don't necessarily have an RSU in all sections of length $d(n)$ of the road. So in this case what upper bounds the distance-limited capacity is the Ad hoc capacity of the network, which has previously been worked out as $\Theta\left(\frac{1}{d}\right)$ and also proved to be an achievable bound in [2].

The second item consists of two cases, which represent the cases where either we are facing single hop communications or where there exists at least one RSU in the transmission range of each vehicle, respectively. In both these cases the achievable upper bound is $\Theta\left(\frac{1}{r_{t}(n)}\right)$, which leads to $\Theta\left(\frac{1}{\ln n}\right)$ as $r_{t}(n)=\Theta(\ln n)$. Note that in the former case, nodes communicate directly in a single hop manner without utilizing RSUs, whereas in the latter case, any two communicating nodes fully rely on RSUs to send their data to each other.

The last item represents the case where we are facing multi-hop communications and we do have at least one RSU in all sections of length $d(n)$ of the road, but at the same time the existence of an RSU in the transmission range, $r_{t}(n)$, of each and every node is not guaranteed. Lets first find an upper bound for the distance limited capacity of the network under these circumstances:

First we divide the road of length $L$ into divisions of length $\frac{n}{R(n)}$ each. In this case, its quite obvious that we can place at least one RSU in each division. So, following the method utilized in [13], we have the following for the upper bound of the distance limited capacity:

$$
n \times \Lambda_{d}^{R}(n) \times \frac{\frac{n}{R(n)}}{r} \leq \frac{n}{r} \rightarrow \Lambda_{d}^{R}(n)=O\left(\frac{R(n)}{n}\right)
$$

To better understand the above relation, take in mind that in this case, for any $d(n)$ distance limited communications, knowing that $d(n) \geq \frac{n}{R(n)}$ and that all vehicles do not have 
immediate access to an RSU, data needs to be sent in ad hoc mode for at most a distance of $\frac{n}{R(n)}$ before it gains access to an RSU. For the achievability, again assume divisions of length $\frac{n}{R(n)}$ of the road, where each of the $R(n)$ RSUs is placed in the middle of each division. We are interested in the maximum number of paths that can pass through an arbitrary section of length $\frac{r_{t}}{2}$. Each vehicle, in order to send its data to a destination, $d(n)$ away from itself, reaches the nearest RSU, which is at most a distance of $\frac{n}{2 R(n)}$ from it. Thus it is clear that a section exactly at the middle of such division, where an RSU is placed, has the most number of paths passing through it. That is, the data of all $\frac{n}{R(n)}$ vehicles within that division has to pass through this section to reach the RSU in that division, Hence the achievability of a throughput of $\Theta\left(\frac{R(n)}{n}\right)$.

To sum up, we emphasize the main ideas behind the proof. There are several factors limiting the capacity, and in each region the dominant factors determine the achievable throughput. The first factor is the capacity of the ad hoc part of the network which is $\Theta\left(\frac{1}{d(n)}\right)$. The capacity due to the RSUs is given by $\Theta\left(\frac{R(n)}{n}\right)$. Also, we should note that capacity higher than $\Theta\left(\frac{n^{n}}{\ln n}\right)$ is not achievable since there is always a section of length $r_{t}(n)$ that has $\Theta(\ln n)$ receiver nodes. These nodes cannot receive at rates higher than $\Theta\left(\frac{1}{\ln n}\right)$.

\section{CONCLUSION}

In this paper we calculated the transport capacity of VANETs utilized in arbitrarily dense downtown road systems. Moreover we introduced a new metric for the capacity of VANETs, in which their specific application, i.e. safety, is accounted for. This is the distance limited capacity, in which nodes need only to communicate with other parties which are within a certain distance from them. Following on, we utilized Road Side Units (RSU)s to see how they affect the VANET capacity in the special case of a single road. Indeed, several simplifying assumptions regarding the mobility models, geometric properties, communication models, and capacity definitions are adopted in this paper. Future work will include extending our results for the single road VANET with infrastructure, to more diverse road topologies, like the grid and general shapes and also developing and analyzing more realistic models.

\section{ACKNOWLEDGEMENT}

The authors would like to thank Daiheng Ni and Aura Ganz for their ever appreciated guidelines.

\section{REFERENCES}

[1] H. Pishro-Nik, A. Ganz and D. Ni, "The Capacity of Vehicular Ad Hoc Networks," 45th Annual Allerton
Conference on Communications, Control and computing, September 2007.

[2] M. Nekoui, H. Pishro-Nik, A. Eslami, "Scaling Laws for Distance Limited Communications in Vehicular Ad hoc Networks," accepted for publication in the proceedings of ICC 2008.

[3] A. Zemlianov and G. de Veciana, "Capacity of Ad Hoc Wireless Networks With Infrastructure Support" IEEE Journal on Selected Areas in Communications, Vol.23, No.3, March 2005.

[4] M. Grossglauser and D. N. C. Tse, Mobility increases the capacity of ad hoc wireless networks, IEEE Transactions on Networking, vol. 10, August 2002. 4

[5] N. Bansal and Z. Liu, Capacity, delay and mobility in wireless ad-hoc networks, in IEEE INFOCOM 2003, March-April 2003.

[6] R. M. de Moraes, H. R. Sadjadpour, and J. J. GarciaLuna-Aceves, On mobility-capacity-delay trade-off in wireless ad hoc networks, IEEE Computer Societys 12th Annual International Symposium on Modeling, Analysis, and Simulation of Computer and Telecommunications Systems (MASCOTS04), pp. 1219, October 2004.

[7] S. N. Diggavi, M. Grossglauser, and D. N. C. Tse, Even one-dimensional mobility increases capacity of wireless networks, IEEE Transactions on Information Theory, vol. 51, November 2005.

[8] M. Garetto, P. Giaccone, and E. Leonardi, On the capacity of ad hoc wireless networks under general node mobility, IEEE INFOCOM, May 2007.

[9] G. B. Folland, Real Analysis: Modern Techniques and Their Applications. John Wiley and Sons Inc., 1999.

[10] D. L. Gerlough, Poisson and traffic: Use of Poisson distribution in highway traffic. Eno Foundation for Highway Traffic Control, 1955.

[11] A. Renyi, On two mathematical models of the traffic on a divided highway, Journal of Applied Probability, pp. 311320, 1964.

[12] R. S. Stravastava, A note on a mathematical model of traffic flow on a divided highway. Transportation Research, 1969.

[13] P. Gupta and P. R. Kumar, The capacity of wireless networks, IEEE Transactions on Information Theory, vol. 46, no. 2, pp. 388404, 2000.

[14] A. Lozano, S. R. Kulkarni, and P. Vishwanath, Throughput scaling in wireless networks with restricted mobility,IEEE International Symposium on Information Theory (ISIT), p. 440, June-July 2004.

[15] J. Mammen and D. Shah, Throughput and delay in random wireless networks with restricted mobility, submitted to IEEE Transactions on Information Theory, August 2005. 